\title{
Suprathreshold contrast perception as a function of spatial frequency
}

\author{
PAUL C. QUINN \\ Brown University, Providence, Rhode Island
}

\begin{abstract}
Several studies have indicated that the exponent of the function relating perceived contrast to physical contrast is influenced by spatial frequency (e.g., Franzen \& Berkley, 1975). This result has been called into question by other studies that have found the exponent to be unaffected by changes in spatial frequency (e.g., Gottesman, Rubin, \& Legge, 1981). Possible artifactual reasons for the spatial frequency effect include failure to take into account range and stimulus repetition effects as well as contrast threshold differences. The present study examined the influence of spatial frequency on the relationship between perceived and physical contrast and controlled for these possible artifacts. Using a modulus-free version of magnitude estimation, judgments of perceived contrast were obtained from 7 observers at each of four spatial frequencies-.75, 4, 15 , and 18 cycles per degree (cpd). When the data were fit with a threshold-corrected power function, an increase in the exponent of the perceived-contrast function was still observed at both low and high spatial frequencies, relative to a middle spatial frequency.
\end{abstract}

Although the influence of spatial frequency on contrast sensitivity has been well documented (for a review, see Sekuler, 1974), its effect on the perception of suprathreshold levels of contrast is currently a matter of debate. A relatively recent concern among visual psychophysicists is how perceived contrast grows as a function of physical contrast for different spatial frequencies. The exponent of the power function relating perceived contrast to physical contrast provides an index of the rate of growth. Franzen and Berkley (1975) initially reported increases in the exponent with increasing spatial frequency (see also Essock, 1982, for a report of high exponents at a high spatial frequency). A number of other investigators have failed to find such an increase, reporting instead an exponent that is independent of spatial frequency (Cannon, 1979; Ginsburg, Cannon, \& Nelson, 1980; Gottesman, Rubin, \& Legge, 1981; Hamerly, Quick, \& Reichert, 1977). Most recently, however, Biondini and Mattiello (1985) reported a new effect of spatial frequency on the perceived-contrast function. Consistent with Franzen and Berkley's (1975) findings, the exponent was found to increase with increases in spatial frequency from an intermediate value. However, in extending Franzen and Berkley's findings, Biondini and Mattiello also found an increase in the exponent with decreases in spatial frequency from an intermediate value.

This research was supported by National Institutes of Health Grant DHHS EY03524 awarded to Stephen Lehmkuhle. The author would like to thank Jon Gottesman, Stephen Lehmkuhle, Scott Stevenson, E. A. Wasserman, and two anonymous reviewers for the many useful suggestions they provided during the preparation of the manuscript, and Pat Anthony for her help in preparing the tables. The author is now with the Department of Psychology, University of Iowa, Iowa City, IA 52242 , and requests for reprints should be sent to this address.
There are several possible procedural differences that might account for the differences in the results of these studies. The first consideration is the range of stimulus contrasts employed. Use of a small range of contrast values has been shown to increase the exponent of the function relating perceived and physical contrast (Cannon, 1984). Thus, the increase in the exponent observed at the highest spatial frequency used by Franzen and Berkley (1975) may reflect the smaller range of stimulus contrasts used at this frequency, rather than a real perceptual difference.

The second consideration is whether the magnitude estimation data are analyzed in threshold-corrected form. Magnitude estimation data are often plotted with a correction for threshold when near-threshold stimuli are used in the stimulus range being scaled (see Engen, 1969). These near-threshold stimuli are associated with a deviation from the simple power function (i.e., a downward curvature or artificial steepening) that has the effect of elevating the exponent. When the data are analyzed with a threshold correction, the deviation is eliminated and the exponent is lowered. It has been shown that failure to take into account differences in contrast threshold at the different spatial frequencies influences the exponent of the function relating perceived and physical contrast (Cannon, 1984; Gottesman et al., 1981). In studies in which increases in the exponent were reported at both low and high spatial frequencies, the data have been analyzed without a threshold correction (Biondini \& Mattiello, 1985; Franzen \& Berkley, 1975). For this reason, the extent to which the observed increases have been due to threshold effects is not known.

The third consideration is the effect of stimulus repetition. Gottesman et al. (1981), over the course of collecting three or four sets of estimations at a single spatial fre- 
quency, found consistent increases in the exponent for single observers. Such an experiential effect could influence the exponent obtained at different spatial frequencies if the frequencies are presented to observers one at a time, as was the case in the Cannon (1979) and Ginsburg et al. (1980) studies. Because of this consideration, it is important to have a single observer scale contrasts at frequencies that are alternated with one another in a single session.

The present investigation was undertaken with these procedural considerations in mind. Its purpose was to determine whether spatial frequency would influence the exponent of the power function relating perceived and physical contrast when the range of contrasts was kept constant and when contrast threshold differences and stimulus repetition effects were taken into account. For this purpose, a modulus-free version of magnitude estimation was employed and observers were asked to judge the perceived contrast for the same range of physical contrasts at each of four spatial frequencies within a single session. The results indicate that an effect of spatial frequency is still obtained even after the data are plotted in thresholdcorrected form.

\section{METHOD}

\section{Subjects}

Seven observers, 6 male and 1 female, served in this experiment. They ranged in age from 21 to 25 . Some of the observers had previous experience in other types of psychophysical tasks, but all were naive as to the purposes of the experiment. All observers reported normal or corrected-to-normal acuity.

\section{Apparatus and Stimuli}

Stationary vertical sine-wave gratings were presented on a Tektronix Model 535 CRT (blue P11 phosphor). A sheet of white cardboard with a central circular aperture was placed in front of the CRT. This cut down the face of the CRT to $3^{\circ}$ of visual angle when it was viewed monocularly from a distance of $85.5 \mathrm{~cm}$. The cardboard was illuminated to match the face of the CRT in all aspects of color. The average luminance of the display was $17 \mathrm{~cd} / \mathrm{m}^{2}$ and the maximum contrast achievable was $61 \%$. The presentation of the gratings was controlled by an Apple computer.

\section{Procedure}

Contrast thresholds. For each observer, contrast thresholds were obtained in a single session for four spatial frequencies-.75, 4 , 15 , and 18 cycles per degree (cpd). Thresholds were measured by the method of adjustment. In order to overcome adaptation effects produced by viewing stationary gratings, the gratings were presented in 5-sec intervals that alternated with 5-sec intervals of a homogeneous field of the same mean luminance as the gratings. The onset and offset of the intervals were signaled by computer-controlled tones. The order of presentation of the different spatial frequencies was randomized for each observer. An observer's mean contrast threshold at each spatial frequency was based on six threshold settings.

Magnitude estimates. In preparation for the magnitude estimation session, suprathreshold contrast levels were fixed for each spatial frequency in steps of $.15, .30, .45, .60, .75$, and $.90 \mathrm{log}$ units above each observer's contrast threshold at that frequency. The modulus-free version of magnitude estimation was employed. Observers were instructed to assign numbers that were proportional to the perceived contrast of the sine-wave grating stimuli. Numerical examples were not provided. At the outset of the magnitude estimation session, the observers were shown a representative range of the physical contrast values they would encounter in the actual experiment. Specifically, a given observer was shown the stimuli that were .30 and $.75 \mathrm{log}$ units above contrast threshold at each spatial frequency. During this preview and during the actual magnitude estimation task, each of the grating stimuli was presented for $5 \mathrm{sec}$, separated by an intertrial interval of approximately $8 \mathrm{sec}$ during which the observer viewed a homogeneous field of the same mean luminance as the gratings. Within a single session, four magnitude estimates were obtained at each of the six contrast levels presented at each spatial frequency. The order in which each combination of spatial frequency and physical contrast was presented was randomized except for the following constraint: all 24 stimuli were presented once before the next repetition of the set. This order of stimulus presentation allowed us to analyze possible stimulus repetition effects.

\section{RESULTS}

Data for the individual observers and for the group as a whole were analyzed. In the analysis of individual data, geometric means were calculated from the four estimates obtained at each combination of physical contrast and spatial frequency. In the analysis of group data, an attempt was made to minimize the intercept variability that arises in a modulus-free magnitude estimation task as a result of interobserver variability. At each spatial frequency, the grand mean of the log values of all the observers' estimates was calculated. The difference between the grand mean and each observer's mean was then calculated and this difference was added to each of the observer's estimates. This procedure has been described by Engen (1969) and is the same one used by Gottesman et al. (1981).

Group data were plotted in two different ways. First, the data were fit with the traditional power function in the form $\log R=n \log C+\log k$, where $R$ is the observer's magnitude estimate, $n$ is the exponent of the power function, $\mathrm{C}$ is the physical contrast of the stimulus, and $\mathrm{k}$ is the gain constant. Second, the data were fit with the threshold-corrected power function suggested by Gottesman et al. (1981). This function takes the form of $\log R=\log \left(C-C_{t}\right)+\log k$, where the added variable $C_{t}$ represents an observer's threshold contrast. Both the power function and threshold-corrected power function provided excellent fits to the data, with $\mathrm{r}^{2}$ values not going below .98 . All of the figures were drawn by computer using a program provided by Hayes (1981).

\section{Group Data-Not Threshold-Corrected}

Group data fitted with the traditional form of the power function are shown in Figure 1, where log magnitude estimate is plotted as a function of log physical contrast for the four spatial frequencies. The log magnitude estimates shown on the $y$-axis are actually arithmetic means computed from the seven individual log magnitude estimates. Similarly, since there were individual differences in the contrast thresholds of the seven observers, the contrast values on the $\mathrm{x}$-axis are arithmetic means of seven different $\log$ physical-contrast values. The functions are dis- 


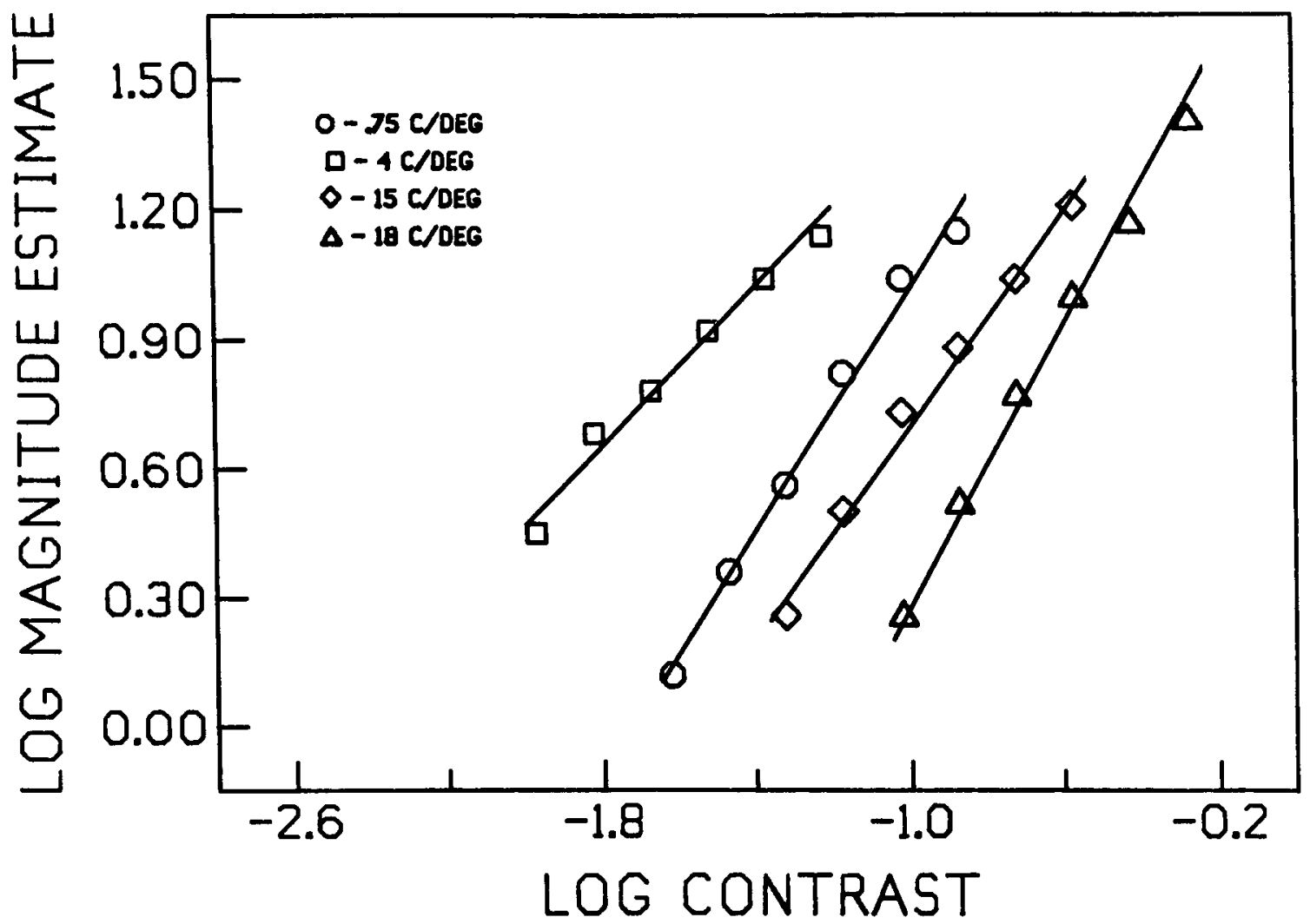

Figure 1. Log magnitude estimate plotted as a function of $\log$ contrast for four different spatial frequencies. Regression lines were fitted by the least squares criterion. Slopes of the regression lines for the different spatial frequencies were as follows: $0.75 \mathrm{cpd}$, slope $=1.42 ; 4 \mathrm{cpd}$, slope $=0.89 ; 15 \mathrm{cpd}$, slope $=1.24 ; 18 \mathrm{cpd}$, slope $=1.51$.

placed along the $x$-axis because of differences in the amount of contrast needed to reach threshold at the different spatial frequencies. The left-to-right ordering of the functions is consistent with the well-established finding that contrast thresholds vary as a function of spatial frequency, with those at intermediate spatial frequencies being lower than those at lower or higher spatial frequencies (see, e.g., Essock \& Lehmkuhle, 1982).

Figure 1 depicts the spatial-frequency-dependent nature of the exponent previously reported by Franzen and Berkley (1975) and Biondini and Mattiello (1985). The exponents were determined by finding the slope of the line of best fit at each spatial frequency as calculated by the method of least squares. There was an increase in the slope of the regression line with increases and decreases in the spatial frequency from the intermediate value of $4 \mathrm{cpd}$. The slope of the regression line increased from 0.89 $(\mathrm{SE}=0.032)$ at $4 \mathrm{cpd}$ to $1.24(\mathrm{SE}=0.032)$ at $15 \mathrm{cpd}$, $1.51(\mathrm{SE}=0.024)$ at $18 \mathrm{cpd}$, and $1.42(\mathrm{SE}=0.043)$ at $.75 \mathrm{cpd}$.

\section{Group Data-Threshold-Corrected}

The effect of fitting the group data with a thresholdcorrected power function is shown in Figure 2. As in Figure 1, the log magnitude estimate plotted on the y-axis is an arithmetic mean calculated from the seven individual $\log$ magnitude estimates. The $\log \left(C-C_{t}\right)$ values on the $\mathrm{x}$-axis were also arithmetic means, calculated from $\log (\mathrm{C}$ $-C_{t}$ ) values obtained for each of the seven observers. Comparison of the exponents of the different thresholdcorrected power functions can be accomplished by calculating the slope of the line of best fit at each spatial frequency. A comparison of Figure 2 with Figure 1 shows that the overall effect of the threshold correction is to lower the slope of the regression line at each spatial frequency. Despite the overall lowering of the slopes, however, the effect of spatial frequency is readily observable. The slope of $0.56(\mathrm{SE}=0.015)$ increases to 0.77 $(\mathrm{SE}=0.022)$ at $15 \mathrm{cpd}, 0.94(\mathrm{SE}=0.041)$ at $18 \mathrm{cpd}$, and $0.89(\mathrm{SE}=0.043)$ at $.75 \mathrm{cpd}$.

\section{Individual Data-Threshold-Corrected}

The influence of spatial frequency on the exponents of the threshold-corrected power function is further documented by the individual-observer data. Table 1 shows the increase in the exponent in each observer's data when an intermediate spatial frequency was changed to higher and lower spatial frequencies. A one-factor analysis of variance, with spatial frequency as a withinsubjects factor, showed that this effect of spatial frequency is indeed significant $[\mathrm{F}(3,18)=16.55, \mathrm{p}<.01]$. Post hoc Tukey HSD tests comparing the mean exponents 


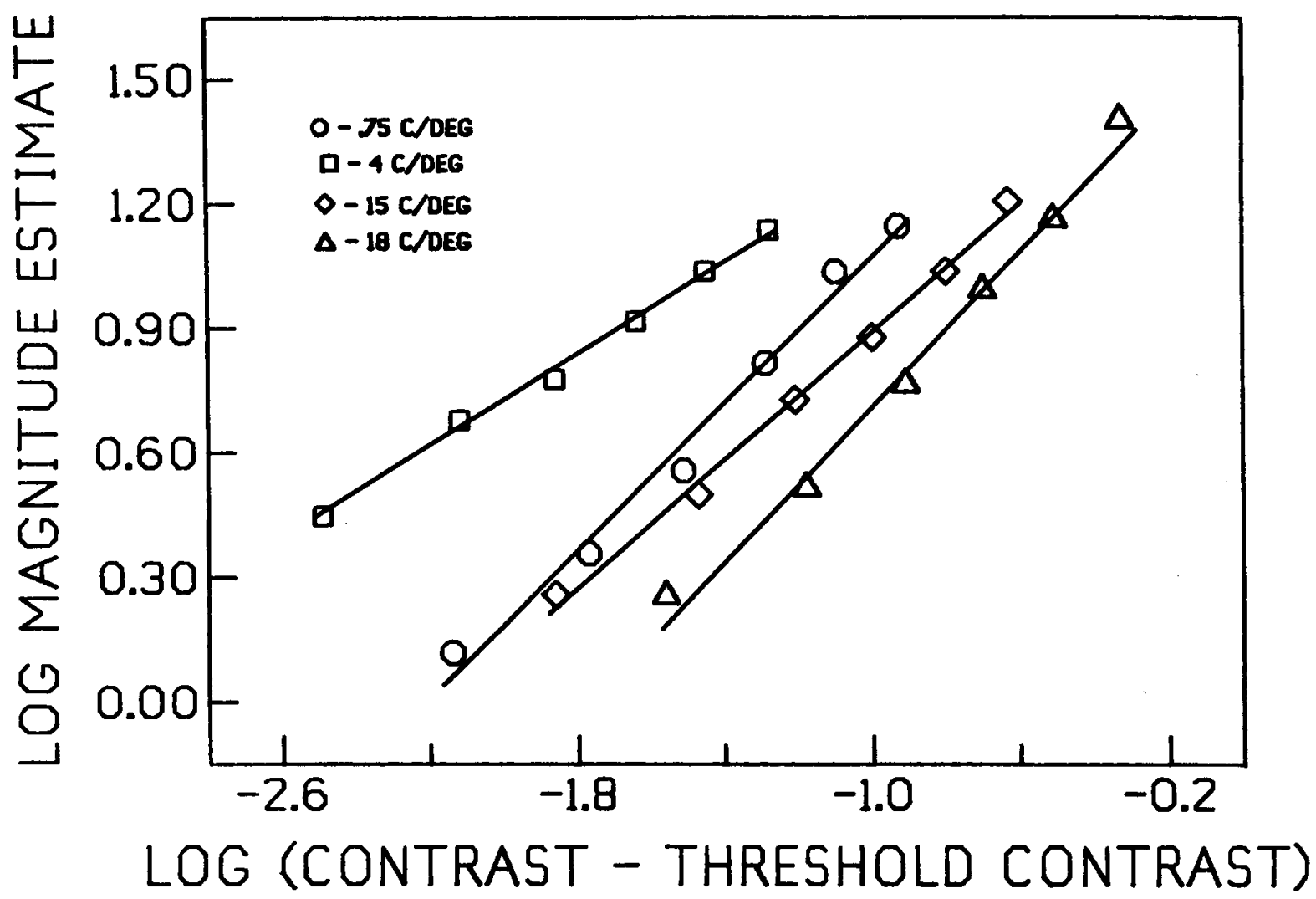

Figure 2. Log magnitude estimate plotted as a function of $\log$ (contrast - threshold contrast) for four different spatial frequencies. Regression lines were fitted by the least squares criterion. Slopes of the regression lines for the different spatial frequencies were as follows: $0.75 \mathrm{cpd}$, slope $=0.89 ; 4 \mathrm{cpd}$, slope $=0.56 ; 15 \mathrm{cpd}$, slope $=0.77 ; 18 \mathrm{cpd}$, slope $=0.93$.

at the different spatial frequencies further revealed the nature of the spatial frequency effect. The mean exponents observed at .75 and $15 \mathrm{cpd}$ were found to be significantly higher than the mean exponent at $4 \mathrm{cpd}$, and the mean exponent at $18 \mathrm{cpd}$ was found to be significantly higher than that at $15 \mathrm{cpd}$ (HSD $=.15, \mathrm{p}<.05$, in each instance). These data thus demonstrate a clear and orderly effect of spatial frequency on the exponent of the threshold-corrected power function relating perceived and physical contrast.

\section{Stimulus Repetition Effects}

Gottesman et al. (1981) reported that the exponent of the threshold-corrected power function relating perceived to physical contrast increased with repeated presentations of a set of contrasts at a single spatial frequency. At $2 \mathrm{cpd}$, for example, they found that the exponent displayed a gradual increase from .58 to .80 over the course of four successive contrast sets. The experimental design employed in the present study allowed for assessment of such repetition effects. Table 2 displays the group exponents for the threshold-corrected power functions for the four successive contrast sets at each spatial frequency. Several findings deserve comment. First, the orderly increase in the exponent with successive contrast sets reported by Gottesman et al. was not observed in the present study. Sec- ond, within a spatial frequency, the range of variation in the exponent was smaller than in the Gottesman et al. study, the largest being .14. Third, it should be noted that for three of four spatial frequencies, the exponent stabilized between the third and fourth presentations of a contrast set. A final observation, of primary importance for the present study, is that the effect of spatial frequency is present in each of the four contrast sets. That is, an increase in the exponent at both high and low spatial frequencies, relative to a middle spatial frequency, can be observed in each contrast set.

\section{Spatial Frequency Anisotropy of Perceived Contrast and Its Dependence on \\ Physical Contrast}

As shown in Figure 1, perceived contrast increased as a function of physical contrast and this increase was more rapid at low and high spatial frequencies than at an intermediate spatial frequency. The data in Figure 1 have been replotted in Figure 3 in an effort to further reveal the nature of the spatial frequency effect. Figure 3 displays $\log$ magnitude estimate as a function of spatial frequency (logarithmically spaced) for the six suprathreshold contrast levels. For the stimuli that are $.15 \log$ units above contrast threshold, the general shape of the contrast sensitivity function can be observed. That is, for the lowest 
Table 1

Threshold-Corrected Power Function Exponents for Individual Observers at the Different Spatial Frequencies

\begin{tabular}{ccccc}
\hline & \multicolumn{4}{c}{ Spatial Frequency (cpd) } \\
\cline { 2 - 5 } Observer & 0.75 & 4 & 15 & 18 \\
\hline 1 & 0.88 & 0.43 & 0.77 & 0.99 \\
2 & 0.75 & 0.74 & 0.80 & 0.94 \\
3 & 0.78 & 0.36 & 0.73 & 0.86 \\
4 & 0.86 & 0.69 & 0.83 & 0.96 \\
5 & 1.07 & 0.57 & 0.62 & 0.80 \\
6 & 1.01 & 0.84 & 0.92 & 1.20 \\
7 & 0.82 & 0.47 & 0.75 & 0.85 \\
Mean & 0.88 & 0.58 & 0.77 & 0.94 \\
\hline
\end{tabular}

Table 2

Exponents of the Threshold-Corrected Power Functions for Successive Contrast Sets at Each Spatial Frequency

\begin{tabular}{cccccc}
\hline & \multicolumn{4}{c}{ Spatial Frequency (cpd) } & \\
\cline { 2 - 5 } Contrast Set & 0.75 & 4 & 15 & 18 & Mean \\
\hline 1 & 0.84 & 0.58 & 0.79 & 0.92 & 0.78 \\
2 & 0.79 & 0.60 & 0.86 & 0.98 & 0.81 \\
3 & 0.92 & 0.52 & 0.72 & 0.87 & 0.76 \\
4 & 0.93 & 0.54 & 0.72 & 1.03 & 0.80 \\
\hline
\end{tabular}

contrast levels, the higher contrast sensitivity observed at intermediate spatial frequencies, as compared with low and high spatial frequencies, is translated into an anisotropy of perceived contrast. As physical contrast is increased, however, the nature of the spatial frequency anisotropy of perceived contrast changes. At .75 log units above contrast threshold, for example, the estimates at .75 and 15 cpd have "caught up" and are equivalent to those at $4 \mathrm{cpd}$; at $18 \mathrm{cpd}$, the perceived contrast is rated even higher than at $4 \mathrm{cpd}$. The latter effect is also observed at $.90 \mathrm{log}$ units above threshold, where perceived contrast for gratings at both 15 and $18 \mathrm{cpd}$ is rated higher than at $4 \mathrm{cpd}$. At higher spatial frequencies and suprathreshold contrast levels, then, the visual system appears to be overcompensating for the lower contrast sensitivity usually observed at these spatial frequencies compared with intermediate frequencies (see, e.g., Essock \& Lehmkuhle, 1982).

\section{DISCUSSION}

The data obtained in the present study indicate that there is an effect of spatial frequency on the exponent of the function relating perceived and physical contrast that is independent of the contrast threshold differences that exist at the different spatial frequencies. Exponents are higher at high and low spatial frequencies than at an intermediate frequency. Furthermore, these exponent differences cannot be attributed to contrast range or stimulus repetition effects. The range of contrasts estimated at each spatial frequency was kept constant and stimulus repetition effects were not observed.

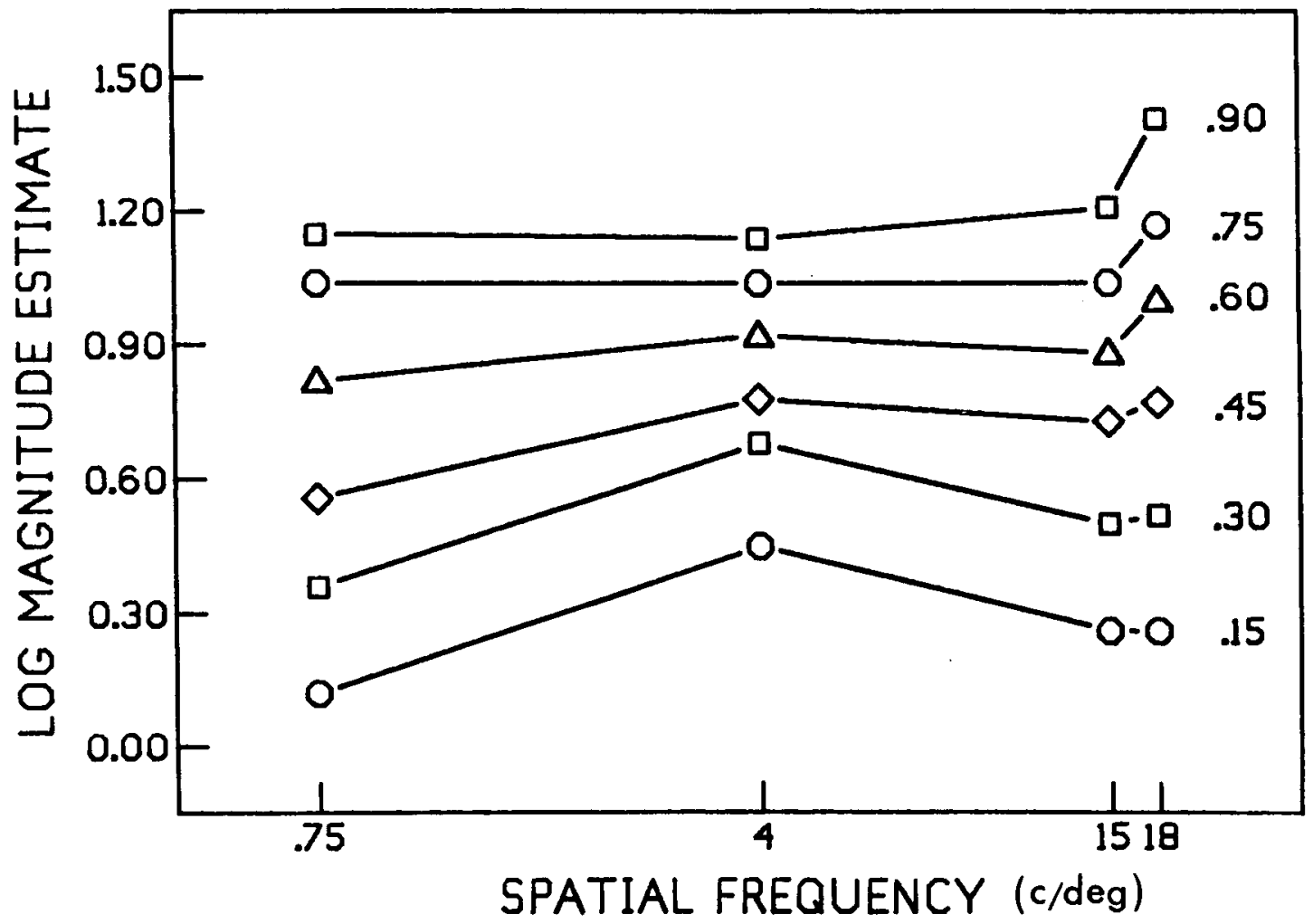

Figure 3. Log magnitude estimate plotted as a function of spatial frequency (logarithmically spaced) for the six different physical contrast levels. Numbers to the right of each of the functions represent the number of $\log$ units above contrast threshold. 


\section{Comparison With Previous Work}

Threshold-corrected data. Two other studies that presented data in threshold-corrected form were those of Gottesman et al. (1981) and Cannon (1984). Gottesman et al. obtained threshold-corrected exponents around .7 for a range of spatial frequencies. However, they did not examine spatial frequencies above $12 \mathrm{cpd}$ and they did observe an increase (although not significant) in the exponent at the lowest spatial frequency tested $(.25 \mathrm{cpd})$. Several points of comparison between the results of the present study and those of Gottesman et al. should be mentioned. First, the .56 value of the exponent obtained at $4 \mathrm{cpd}$ in the present study matches well with the range of exponents observed at 2 cpd by Gottesman et al. Second, the present results extend those of Gottesman et al. in that they indicate an increase in the threshold-corrected exponent at spatial frequencies larger than $12 \mathrm{cpd}$. Finally, at the lowest spatial frequency examined in the present study, an increase in the exponent was observed and, in this case, was found to be reliable. Thus, the present results are in good qualitative agreement with those of Gottesman et al.

Recently, Cannon (1984) had observers scale a similar range of physical contrasts at 2 and $16 \mathrm{cpd}$ and reported equivalent threshold-corrected exponents at these frequencies. On the basis of these data, Cannon concluded that once possible artifacts (i.e., smaller range of stimulus contrasts, threshold effects) are controlled for, spatial frequency effects on the exponent do not appear. At first glance, the data obtained in the present study do not appear to be consistent with those obtained by Cannon. However, recall that in the present study, increases in the mean exponents were obtained when spatial frequency was changed from 4 to $.75 \mathrm{cpd}$ and also when it was changed from 15 to $18 \mathrm{cpd}$ (see Table 1). Therefore, it is possible (and indeed likely, given the observed trends) that at least a rough equivalence of exponents would have been obtained at 2 and $16 \mathrm{cpd}$, had these frequencies been tested. In view of the pattern of results obtained here, and given the small number of data points on the spatial frequency continuum in Cannon's study, it appears that spatial frequency effects on the exponent can be obtained if one tests with a wide range of spatial frequencies.

Threshold-uncorrected data. The uncorrected exponents obtained in the present study confirm the previously reported increase in the exponent as spatial frequency is increased and decreased from an intermediate value (Biondini \& Mattiello, 1985; Franzen \& Berkley, 1975). Another worthwhile comparison is that between the exponents obtained at 15 and $18 \mathrm{cpd}$ in the present study and those reported by Essock (1982) at $16 \mathrm{cpd}$. This comparison seems especially noteworthy inasmuch as the same apparatus was used in both studies. Essock reported exponents of 1.33 and 1.90 , slightly higher than those reported here. However, the range he used was slightly smaller (.60 log units) than that used here $(.75 \log$ units). Because a smaller range of stimulus contrasts would be expected to elevate the exponent, the exponents reported here seem to be in good agreement with those of Essock. Although the effects of spatial frequency on the uncorrected exponents found in the present study do appear to be consistent with those reported by Franzen and Berkley (1975) and Biondini and Mattiello (1985), they conflict with earlier reports that have shown the uncorrected exponent to be independent of spatial frequency (Cannon, 1979; Hamerly et al., 1977). Although the effects of spatial frequency reported in previous studies may be explained in part by threshold and range effects, the effects found in the present study cannot be so explained. One salient difference between the procedure used here and those of Hamerly et al. and Cannon is the use of a withininstead of between-subjects design in the present study. It is possible that the within-subjects design, in allowing individual observers to directly compare the growth of perceived contrast at different spatial frequencies in a single session, may be a more sensitive index of variations in the exponent at different frequencies. This suggestion is indirectly supported by some earlier findings in the animal learning literature on stimulus generalization. In the classic generalization experiment, subjects are trained to respond to one value along a stimulus dimension and are then tested for generalization to other values along that dimension. A within-subjects design, in which each subject is tested on all the different values along a sensory dimension (e.g., light intensity), is more likely to yield differential responding than is a between-subjects design, in which each subject is tested on only one value along the dimension (Donahoe, McCroskery, \& Richardson, 1970). Future work on the perceived-contrast function at different spatial frequencies, comparing a withinsubjects design with a between-subjects design using the same apparatus and procedure, should prove informative.

\section{Anisotropy of Perceived Contrast at High Spatial Frequencies}

At high physical-contrast values, the faster growth of perceived contrast as a function of physical contrast at the extreme spatial frequencies resulted in a perceivedcontrast equivalence at low and intermediate spatial frequencies; at high spatial frequencies, perceived contrast surpassed that at an intermediate spatial frequency (see Figure 3). These observations suggest that as physical contrast is increased at low spatial frequencies, the visual system appears to compensate for the high contrast threshold usually reported at these frequencies. Such a compensation mechanism would allow for perceivedcontrast constancy across low and middle spatial frequencies at high suprathreshold levels of contrast. As physical contrast is increased at high spatial frequencies, on the other hand, the visual system appears to overcompensate for the high contrast threshold that is normally observed at these frequencies. Such an overcompensation mechanism would allow perceived contrast at high spatial frequencies to be higher than perceived contrast at 
lower frequencies at high physical contrasts. One might ask why an overcompensation mechanism is present at high spatial frequencies. A speculative answer is that the high-spatial-frequency result reflects the visual system's way of enhancing high-contrast edge information. Highcontrast edges that "stand out" in a visual scene and define borders of objects could be especially useful information in the fundamental perceptual task of separating figure from ground.

\section{REFERENCES}

Biondini, A. R., \& Mattiello, M. L. F. (1985). Suprathreshold contrast perception at different luminance levels. Vision Research, 25, 1-9. CanNon, M. W. (1979). Contrast sensation: A linear function of stimulus contrast. Vision Research, 19, 1045-1052.

Cannon, M. W. (1984). A study of stimulus range effects in free modulus magnitude estimation of contrast. Vision Research, 24, 1049-1055.

Donahoe, J. W., McCroskery, J. H., \& Richardson, W. K. (1970). Effects of context on the postdiscrimination gradient of stimulus generalization. Joumal of Experimental Psychology, 84, 58-63.
Engen, T. (1969). Psychophysics II. Scaling methods. In J. W. Kling \& L. A. Riggs (Eds.), Woodworth and Schlosberg's experimental psychology: Vol. I. Sensation and perception (3rd ed.). New York: Holt, Rinehart \& Winston.

Essock, E. A. (1982). Anisotropies of perceived contrast and detection speed. Vision Research, 22, 1185-1192.

Essock, E. A., \& LeHMKuHLe, S. (1982). The oblique effects of pattern and flicker sensitivities: Implications for mixed physiological input. Perception, 11, 441-445.

Franzen, O., \& BERKLEY, M. (1975). Apparent contrast as a function of modulation depth and spatial frequency. Vision Research, 15, $655-660$.

Ginsburg, P., CAnnon, M., \& Nelson, M. (1980). Suprathreshold processing of complex visual stimuli: Evidence for linearity in contrast perception. Science, 208, 619-621.

Gottesman, J., Rubin, G., \& LegGe, G. (1981). A power law for perceived contrast in human vision. Vision Research, 21, 791-799.

Hamerly, J. R., Quick, R. F., \& ReicherT, T. A. (1977). A study of grating contrast judgment. Vision Research, 17, 201-207.

HAYES, W. (1981). GRAPH: A BASIC-11 graphics program for digital plotters. Behavior Research Methods \& Instrumentation, 13, 367 SekUler, R. (1974). Spatial vision. Annual Review of Psychology, 25, 195-232. 